\title{
TRATAMENTO DA HIPERTENSÄO ARTERIAL - RESPOSTAS DE MÉDICOS BRASILEIROS A UM INQUÉRITO
}

*D. Mion Jr, A.M. G. Pierin, A. Guimarães

Trabalho realizado na Liga de Hipertensão do Hospital das Clínicas da Faculdade de Medicina da USP; Instituto Central do Hospital das Clínicas Faculdade de Medicina da Universidade de São Paulo, SP.

RESUMO - 0 III Consenso Brasileiro de Hipertensão Arterial recomenda o uso de tratamento farmacológico (TF) e não-farmacológico (TNF). Em nosso meio não se tem conhecimento como esta recomendação vem sendo seguida pelos médicos.

OBjetIvos. a) caracterizar o TNF quanto à indicação e aceitação pelos hipertensos; b) caracterizar o TF quanto ao critério de escolha e drogas prescritas; e c) identificar a opinião do médico sobre o grau de adesão do paciente ao TF e TNF.

Métodos. Foram enviados 37.904 questionários com cartaresposta por mala direta para médicos brasileiros e recebidas 2.519 respostas (57\% região Sudeste, $41 \%$ cardiologistas e $26 \%$ clínicos).

Resultados. I. $62 \%$ dos médicos que responderam recomendam TNF para 25\% dos pacientes. 2- Os TNF mais recomendados são: dieta hipossódica (55\%), perda de peso $(29 \%)$ e prática de exercícios (8\%), sendo que os médicos acreditam que $50 \%$ dos pacientes seguem dieta hipossódica, $20 \%$ prática de exercícios e $19 \%$ perda de peso. 3- 0 TF é iniciado com o uso de droga isolada (88\%) e quando não há controle da pressão arterial $55 \%$ indicam associação de outras drogas e $33 \%$ aumentam a dose. 4- As drogas mais utilizadas são diuréticos $(53 \%)$ e inibidores da ECA (24\%). 5- Os principais critérios para escolha das drogas são experiência pessoal (32\%) e características do paciente (31\%). 6 Os médicos $(60 \%)$ acreditam que a adesão ao TF é melhor.

Conclusão. As recomendações do III Consenso Brasileiro de Hipertensão Arterial estão sendo seguidas parcialmente para 0 TNF e dentro do esperado para o TF.

UNITERMOS: Hipertensão arterial. Tratamento farmacológico. Tratamento não-farmacológico. Hipertensão no Brasil.

\section{INTRODUÇÃO}

A hipertensão arterial é uma doença altamente prevalente em nosso meio, atingindo cerca de 15 a 20\% da população adulta com mais de 18 anos, chegando aíndices de $50 \%$ nas pessoas idosas' . Assim, é fundamental investigar como os médicos brasileiros estão tratando a hipertensão arterial, tanto do ponto de vista farmacológico como não-farmacológico, com o intuito de orientar a difusão de conhecimentos e reduzir os altos índices de morbidade e mortalidade que a doença acarreta.

*Correspondência: Décio Mion J r.

Av. Dr. Eneas Carvalho de Aguiar, 255 - 7 o andar sala 7032 - Instituto Central do Hospital das Clínicas Faculdade de Medicina da Universidade de São Paulo

Cep: 05403-000 - São Paulo - Caixa Postal 8091 Tel/Fax. (11) 3082-2659
O III Consenso Brasileiro de Hipertensão Arterial², distribuído e divulgado para toda comunidade médica brasileira, recomenda que o tratamento do paciente hipertenso deve ser instituído quando os níveis de pressão arterial são iguais ou superiores a | 40/90 mm Hg. Recomenda-se para hipertensos leves, caracterizados por diastólica entre 90-99 e sistólica entre | 40- 159 $\mathrm{mm} \mathrm{Hg}$, tratamento não-farmacológico isolado durante 12 meses para pacientes do grupo de risco "A", que não apresentam fatores de risco e nem lesões de órgãosalvo e durante 6 meses para pacientes do grupo de risco "B", que apresentam fatores de risco, exceto diabetes melito, mas não apresentam lesões de órgãos-alvo. Caso não haja controle ao final deste período, deve ser associado tratamento farmacológico. Para pacientes do grupo de risco "C", que apresentam lesões de órgãosalvo ou doença cardiovascular clinicamente identificável e/ou diabete melito, é recomendado tratamento farmacológico imediato, além do não-farmacológico.

Ainda de acordo com o III Consenso Brasileiro de Hipertensão Arterial ${ }^{2}$, as medidas não-farmacológicas que apresentam eficácia comprovada em reduzir a pressão arterial são redução do peso corporal, da ingestão de sal e do consumo de bebidas alcoólicas e prática regular de exercícios físicos. Do ponto de vista farmacológico, é recomendada, no início do tratamento para hipertensos leves, uma droga pertencente a uma das 6 classes de anti-hipertensivos, a 
saber: diuréticos, betabloqueadores, simpatolíticos de ação central, antagonistas dos canais de cálcio, inibidores da enzima conversora da angiotensina e antagonistas do receptor da angiotensina II. Quando não há controle da pressão arterial com monoterapia ou surgem efeitos adversos, pode-se aumentar a dose da droga em uso, adicionar uma segunda droga ou substituir a monoterapia.

Apesar do progresso alcançado pela indústria farmacêutica na formulação de medicamentos eficazes e com baixos índices de efeitos indesejáveis, o controle da doença nem sempre é adequado. A falha no seguimento do tratamento proposto, caracterizando falta de adesão e conseqüente controle inadequado da pressão arterial é freqüente e está associado a vários fatores. Em relação ao tratamento medicamentoso, efeitos indesejáveis das drogas e custo têm sido apontados como elementos importantes. O tratamento não-farmacológico, por outro lado, tem falhado freqüentemente no seguimento de longo prazo pela falta de constância e persistência dos pacientes. Considerando estes aspectos, a escolha do tratamento anti-hipertensivo, incluindo as medidas farmacológicas e não-farmacológicas, deve merecer atenção especial por parte dos membros da equipe de saúde.

Face à existência de recomendações sobre o tratamento da hipertensão emitidas pelo III Consenso Brasileiro de Hipertensão Arterial$^{2}$ e ausência de dados sobre a conduta real dos médicos na prática médica diária, o presente estudo teve como objetivos: a) identificar a porcentagem de pacientes que recebe tratamento não-farmacológico, quais são as medidas não-farmacológicas mais recomendadas pelos médicos e aquelas que eles acreditam que os pacientes seguem mais freqüentemente; b) caracterizar as drogas isoladas ou em associação, fixa ou não, prescritas no tratamento farmacológico, e identificar os critérios adotados pelo médico na escolha das mesmas; c) identificar a opinião do médico sobre o grau de adesão do paciente ao tratamento farmacológico e não-farmacológico.

\section{Métodos}

Foram enviados 37.904 questionários com carta-resposta por mala direta para médicos brasileiros pertencentes ao painel de médicos do Laboratório Novartis. O questionário continha | | questões relacionadas aos objetivos do estudo descritas a seguir. a) Dados de identificação: incluindo sexo, especialidade médica, ano de formatura e estado de origem.

b) Caracterização da clientela atendida, incluindo percentual de hipertensos atendidos/dia e gravidade da hipertensão classificada como leve (sistólica $=$ | 40- I 59 e/ou diastólica $=90-99 \mathrm{~mm} \mathrm{Hg}$ ), moderada (sistólica $=160-179$ e/ou diastólica $=100$ 109 mm Hg) e grave (sistólica $\geq 180$ e/ou diastólica $\geq 110 \mathrm{~mm} \mathrm{Hg}$ ).

c) Identificação da porcentagem de pacientes que recebe orientação para tratamento não-farmacológico, quais são as medidas não-farmacológicas mais recomendadas pelos médicos e quais as medidas que os médicos acreditam que os pacientes seguem mais freqüentemente.

d) Caracterização das drogas prescritas em ordem de preferência do médico e identificação dos critérios de escolha do tratamento anti-hipertensivo, dentre I I opções para tratamento farmacológico inicial:

1. experiência pessoal com a droga;

2. freqüência de efeitos colaterais da droga;

3. credibilidade do laboratório fabricante;

4. características do paciente;

5. custo da droga;

6. relacionamento com o representante do laboratório fabricante;

7. sugestão de colega que teve bons resultados com a droga;

8. ouvir apresentação de especialistas sobre as características da droga;
9. eficácia da droga;

10. aprovação da droga pelo FDA-EUA;

I I. droga nova;

e 6 opções para associação fixa de antihipertensivos:

I. eficácia das drogas em associação;

2. emprego de doses baixas dos medicamentos;

3. favorecer a adesão ao tratamento;

4. maior comodidade posológica;

5. menor freqüência de efeitos colaterais; 6. potencializar ação de uma das drogas; além da identificação da conduta dos médicos quando não há controle com monoterapia; e 7. identificação da opinião do médico sobre o grau de adesão do paciente ao tratamento farmacológico e não-farmacológico.

\section{Resultados}

Foram recebidas 2.519 cartas-respostas, correspondentes a $6,6 \%$ do total enviado. De acordo com a procedência, 57\% das respostas eram da região sudeste, $23 \%$ da região sul, 13\% da região nordeste e o restante das demais regiões. Dos médicos que responderam ao questionário, $80 \%$ eram do sexo masculino e $76 \%$ eram graduados nas décadas de 70 e 80, com especialidade em cardiologia (41\%), clínica médica $(26 \%)$ ou geriatria (14\%).

Em relação ao número de pacientes hipertensos atendidos/dia, 47\% dos médicos informaram que $50 \%$ ou mais dos atendimentos são de pacientes hipertensos

Quanto à gravidade da doença, os médicos responderam que $83 \%$ dos pacientes atendidos eram hipertensos leves (45\%) ou moderados (38\%) e somente 17\% eram graves

\section{Medidas não-farmacológicas}

A maioria (62\%) dos médicos informou que recomenda o tratamento não-farmacológico para 25\% dos pacientes e só 17\% recomendam para todos pacientes (Figura I).

As medidas não-farmacológicas mais recomendadas pelos médicos foram dieta 
hipossódica (55\%) e perda de peso (29\%), enquanto a prática de exercícios físicos foi recomendada por apenas 8\% (Figura 2).

As medidas não-farmacológicas mais freqüentemente seguidas pelos pacientes, de acordo com a opinião dos respondedores, foram dieta hipossódica (50\%), prática de exercícios físicos (20\%) e perda de peso (19\%).

Tratamento farmacológico

As drogas mais recomendadas pelos médicos foram diuréticos (53\%) e inibidor da ECA (24\%) (Figura 3).

O tratamento farmacológico de hipertensos leves e moderados é iniciado com o uso de droga isolada em $88 \%$ das vezes (Figura 4).

Quando não há controle da pressão arterial com o emprego de monoterapia, cerca da metade dos médicos (55\%) indicou que associa drogas de diferentes mecanismos de ação e 33\% aumentam a dose da droga em uso (Figura 5).

As associações de drogas mais freqüentemente usadas foram: a) inibidor da ECA + diurético (46\%); b) betabloqueador + diurético (34\%); e c) droga de ação central + diurético (17\%). Nas associações, 30\% responderam que são fixas e $13 \%$ não fixas.

Os aspectos que os médicos consideram mais importantes na escolha da droga para o tratamento inicial dos hipertensos leves/moderados foram: a) experiência pessoal com a droga (32\%); b) características do paciente (3 I\%); e c) eficácia da droga (20\%) (Figura 6).

Os aspectos considerados mais importantes na escolha de associação fixa de drogas anti-hipertensivas foram: a) eficácia das drogas em associação (56\%); b) emprego de doses baixas dos medicamentos (14\%); c) favorecer adesão ao tratamento (13\%).

Adesão do paciente ao tratamento farmacológico e não-farmacológico

A maioria (60\%) dos médicos acredita que há maior adesão ao tratamento farma-
Figura I - Distribuição percentual das respostas dos médicos em relação ao percentual de pacientes que eles orientam para tratamento não-farmacológico. São Paulo, 2000 ( $n=2474)$.

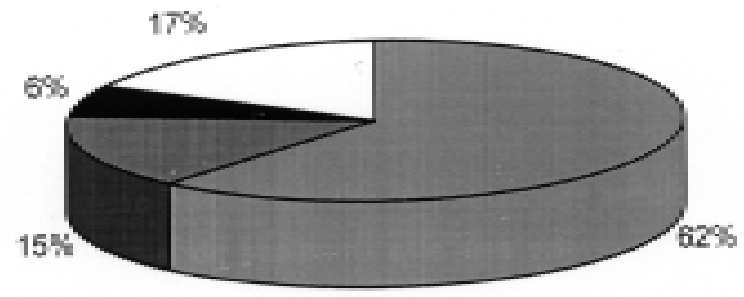

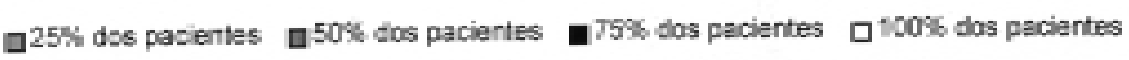

Figura 2- Percentual das respostas mais assinaladas pelos médicos quanto à orientação das medidas não-farmacológicas para hipertensos leves e moderados. São Paulo, 2000 (n=2434).

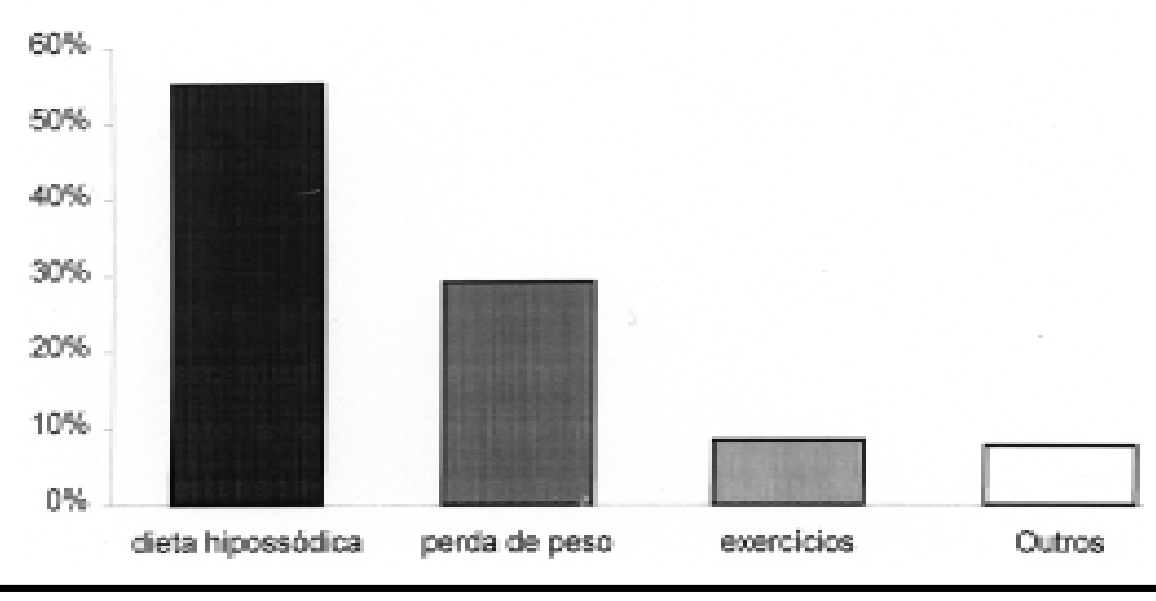

Figura 3 - Percentual das respostas dos médicos quanto às classes de drogas mais prescritas para o tratamento de hipertensos leves e moderados. São Paulo. ( $n=2443)$.

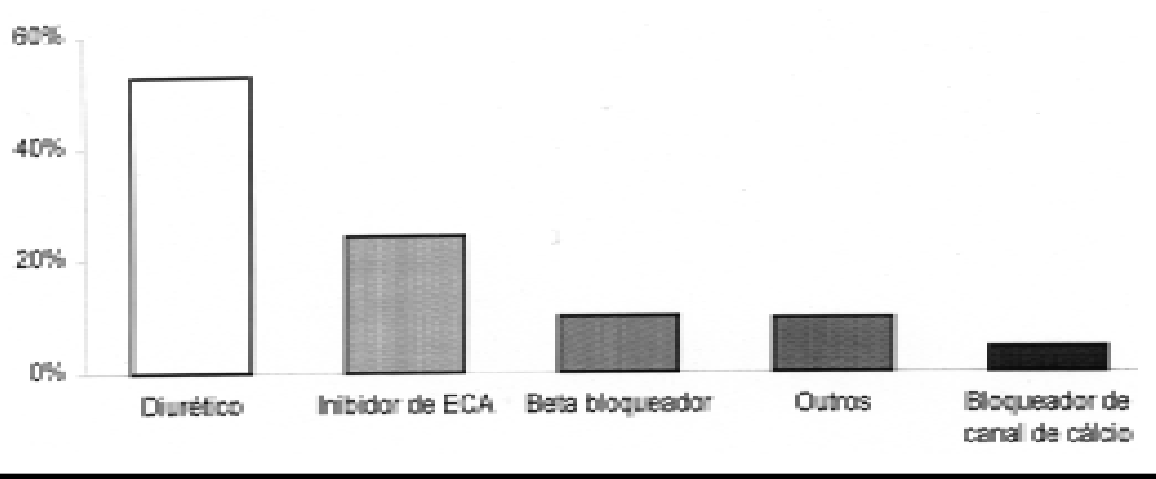


cológico do que ao tratamento não-farmacológico (8\%), considerando-os isoladamente. A adesão foi considerada boa para ambos os tipos de tratamento por $19 \%$ dos médicos e ruim, concomitantemente para ambos, por 13\% (Figura 7).

\section{Discussão}

O número de respostas analisadas correspondente a $6,6 \%$ dos questionários enviados e representa resposta satisfatória para este tipo de pesquisa. No entanto, uma das limitações deste estudo foi a ausência de amostragem da população de todos os médicos brasileiros.

Os dados obtidos são mais representativos de médicos do sexo masculino, da região sudeste e com especialidade em cardiologia, na qual há intensa programação de educação continuada na área de hipertensão. Graduados entre 1970 e 1980 e com grande clientela de hipertensos (cerca de $50 \%$ dos pacientes), estes respondedores devem estar motivados a acompanhar a dinâmica do conhecimento nessa área.

A maioria dos respondedores (62\%) só indicava tratamento não-farmacológico para um quarto dos seus pacientes. Porém, se aumentarmos o ponto de corte para a indicação em, pelo menos $50 \%$ dos pacientes, o percentual de respondedores sobe para $77 \%$, enquanto apenas uma pequena minoria ( $17 \%)$ indicava este tipo de tratamento em $100 \%$ dos seus pacientes, como preconizado pelo III Consenso Brasileiro de Hipertensão Arterial, divulgado no ano de 1998. Este achado é preocupante e sugere algumas possibilidades como divulgação insuficiente das recomendações, mensagem transmitida de modo inadequado ou dificuldades dos profissionais na sua aplicação. Todavia, os que prescreviam este tipo de tratamento, indicavam as principais medidas (dieta hipossódica, perda de peso e prática regular de exercício físico), sugerindo que, das três possibilidades aventadas para a au-
Figura 4 - Percentual das respostas dos médicos quanto à conduta ao iniciar 0 tratamento de hipertensos leves e moderados. São Paulo, 2000 ( $n=2493)$.

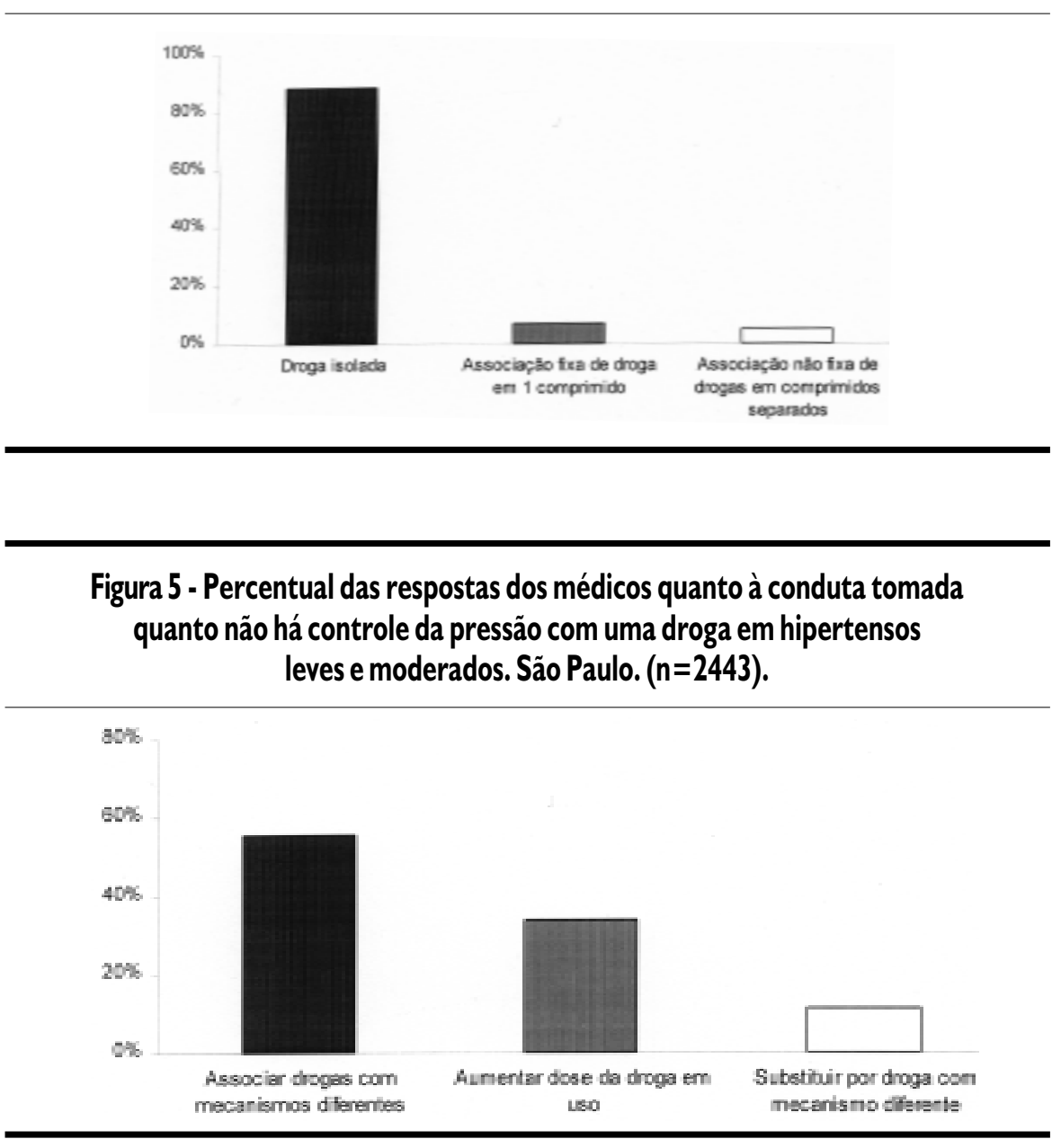

Figura 6 - Distribuição percentual das respoastas dos médicos quanto aos aspectos citados na escolha de uma droga para o tratamento de hipertensos leves e moderados. SãoPaulo. ( $n=2443)$.

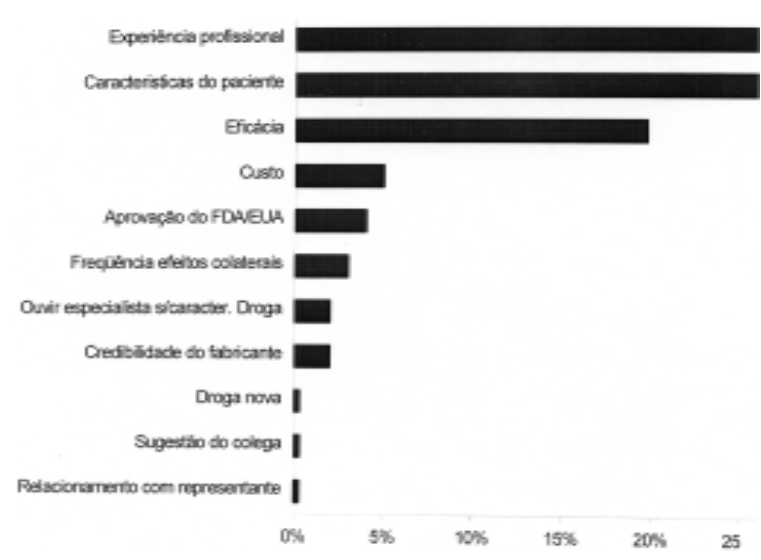


sência de uma aplicação generalizada a todos os hipertensos, a primeira e a última sejam as mais prováveis.

Aanálise dos dados relativos ao tratamento farmacológico também revelou dados que merecem destaque. No que se refere às drogas escolhidas pelos médicos para o tratamento inicial de hipertensos leves, os diuréticos foram as mais citadas na primeira escolha (38\%) seguidas pelos inibidores da enzima de conversão, betabloqueadores ebloqueadores de canais de cálcio. Estes dados estão em acordo com as recomendações do III Consenso Brasileiro de Hipertensão Arterial ${ }^{2}$ e também refletem a preocupação dos médicos em relação ao custo da medicação, dando preferência aos diuréticos, drogas com o custo mais baixo dentre os anti-hipertensivos. Também Akashi e colaboradores verificaram que dos I4I pacientes selecionados que procuraram hospital terciário com diagnóstico prévio de hipertensão, 76\% relatavam estar sob uso de anti-hipertensivos, sendo o medicamento mais referido pelos pacientes os diuréticos tiazídicos. Diferentemente dos nossos resultados, a seqüência observada por estes autores foimetildopa, inibidores da enzima conversora da angiotensina, bloqueadores de canais de cálcio e, finalmente, betabloqueadores ${ }^{3}$. Nos Estados Unidos, a tendência de uso de inibidor de enzima conversora da angiotensina e bloqueadores dos canais de cálcio é verificada na prática apesar do VI Joint National Committee orientar para o uso de diuréticos e betabloqueadores no início do tratamento 4.5 .

A grande maioria dos respondedores (88\%) inicia o tratamento com monoterapia, contrariando a tendência atual de associar drogas para controlar melhor a pressão arterial e facilitar a adesão do paciente ao tratamento. $\mathrm{Na}$ escolha das associações, as duas mais freqüentemente citadas foram diurético com inibidor da ECA e diurético com betabloqueador. No estudo de Akashi et al. ${ }^{3}$ a associação com tiazídicos seguiu a mesma sequência observada com

\section{Figura 7 - Distribuição percentual das respostas dos médicos quanto à opinião sobre a adesão aos tratamentos farmacológico e não-farmacológico. São Paulo. ( $n=2443)$.}

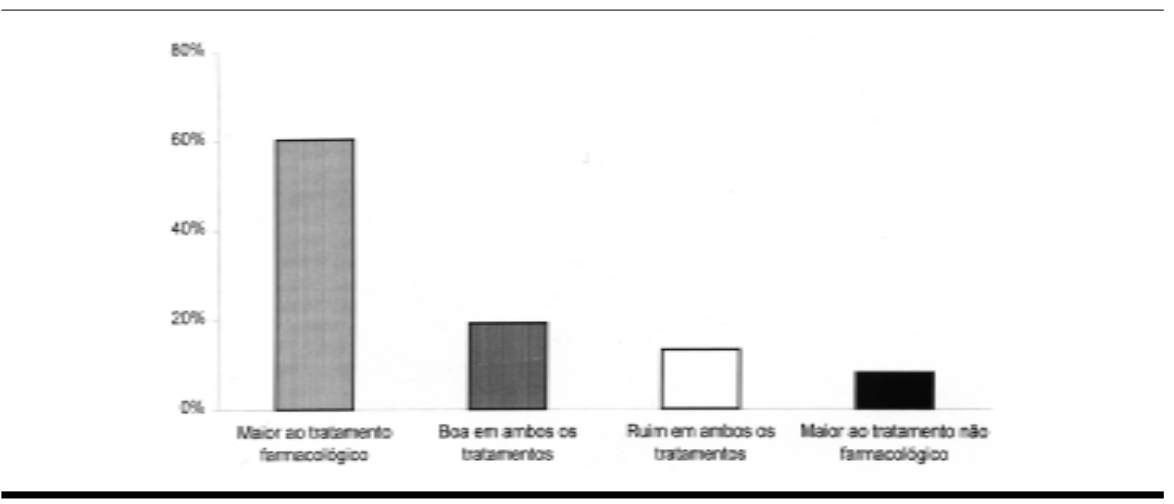

as drogas isoladas: metildopa, inibidores da enzima conversora da angiotensina, bloqueadores dos canais de cácio, propranolol e prazosin.

Dentre os fatores importantes na escoIha das drogas, o item mais citado foi a experiência pessoal com a droga, seguido das características do paciente e eficácia das drogas. Curiosamente, alguns aspectos vistos como importantes, tais como custo e freqüência de efeitos colaterais, receberam pouca prioridade, estando entre os menos citados, $6 \%$ e $4 \%$ dos respondedores, respectivamente. Além destes, outros aspectos poucos citados na escolha da droga inicial e que merecem destaque foram, em ordem decrescente, aprovação pelo FDA (EUA), credibilidade do fabricante, droga nova e relacionamento com representantes, todos três instrumentos habituais de propaganda. $\mathrm{Na}$ escolha de associação fixa de drogas antihipertensivas $\mathrm{o}$ aspecto mais importante ressaltado foi eficácia das drogas em associação. Favorecer adesão ao tratamento esteve em terceiro lugar de importância, abaixo de emprego de doses baixas dos medicamentos.

O tratamento farmacológico foi considerado pela maioria como aquele que promove maior adesão ao tratamento. No entanto, mesmo dados norte-americanos evidenciam que somente $27 \%$ dos pacientes estão com pressão abaixo de 1 40/90 mm
$\mathrm{H} g^{5}$. Como a maior parte destes pacientes deve estar sob tratamento farmacológico, não parece claro que tomar remédios seja mais fácil do que seguir mudanças de hábitos de vida ou que, talvez, as duas modalidades de tratamento sejam igualmente difíceis de serem seguidas. A adesão ao tratamento sofre influência de variáveis relativas ao paciente, tais como idade, sexo, raça, nível socioeconômico, escolaridade, hábitos de vida, aspectos culturais, crenças de saúde, dentre outras. Homens, pessoas mais jovens e com baixa escolaridade tendem a ser menos aderentes ao tratamento. A condição socioeconômica insatisfatória dificulta o acesso ao tratamento e aquisição das medicações. Estudo realizado em nosso meio mostrou que, em relação aos remédios, os motivos que mais contribuíram para que os pacientes deixassem de tomá-los foram custo, ter que tomar várias vezes ao dia e efeitos indesejáveis das drogas ${ }^{6}$.

Portanto, os dados do presente estudo, apesar da limitação do número de respostas, sugerem que os médicos estão seguindo monoterapia recomendações do III Consenso Brasileiro de Hipertensão Arterial ${ }^{2}$ estão sendo seguidas principalmente no que diz respeito à escolha das drogas, apesar de forma distinta entre as diferentes classes farmacológicas, e que o tratamento 
não-farmacológico está sendo indicado para pequeno número de pacientes, talvez porque os médicos assinalaram que a adesão é melhor com tratamento farmacológico. No entanto, outros estudos, com metodologias mais adequadas, precisam ser desenvolvidos para tentar elucidar ainda mais estes importantes aspectos da terapêutica da hipertensão arterial.

\section{Agradecimentos}

Agradecemos a todos colegas que nos auxiliaram na realização deste estudo preenchendo e enviando o questionário. Agradecemos também a Novartis Biociências S.A. pela colaboração na realização deste estudo.

\section{SUMMARY}

\section{Survey about Hypertension TREATMENT}

INTRODUCTION. The third Brazilian Consensus of Hypertension recomends the usage of pharmacological (PT) and nonpharmacological treatment (NPT). In Brazil, we don't know how if this recommedation has been followet by doctors.

OBIECTIVES, a) to characterize NPT regarding prescription and acceptability by hypertensive patients; b) characterize PT concerning the criterion used for choosing medication and medication prescribed; and c) identify doctors' opinions about patients' degree of acceptability to PT and NPT.

METHODS. 37904 questionnaires with reply-paid envelopes were sent to Brazilian doctors and 2519 of those were replied (57\% from the Southeast region, 41\% were cardiologists, and $26 \%$ clinicians).

RESULTS. 1- $62 \%$ of doctors recommend NPT to $25 \%$ of their patients. 2 - The most recommended NP treatments are: hyposodic diet (55\%), weight reduction (29\%) and physical exercises (8\%), considering that doctors believe that $50 \%$ of their patients follow a hyposodic diet, 20\% do physical exercises and 19\% lose weight. 3-Only one medication isprescribed at the beginningofaPT (88\%) and when blood pressure isn'tunder control, 55\% of the doctors associate other medications with it whereas $33 \%$ of them increase the dose. 4 - The most prescribed medications are: diuretics (53\%) and ECAinhibitors (24\%). 5 - The main criteria for choosing medication are: personal experience (32\%) and characteristics of patients (3/\%). 6 - Doctors (60\%) believe that acceptability to PT is better.

CONCLUSION. The recommendations of the Third Brazilian Consensus of Hypertension have been followed partially con- cerning NPT and as expected regarding PT. [Rev Ass Med Brasil 200 I; 47(3): 249-54]

KEY wORDS: Hypertension. Pharmacologial Treatment. Non-pharmacologial treatment.

\section{RefERÊNCIAS}

I. Duncan BB, Schmidt IM, Achutti AP, Polanczyk CA, Benia LR, Maia AA. Socioeconomic distribuition of noncommunicable disease risk factors in urban Brazil: the case of Porto Alegre. Bull PAHO 1993; 27:337-49.

2. III Consenso Brasileiro de Hipertensão Arterial. Rev Clín Terap 1998; 24:231-72.

3. Akashi D, Issa FK, Pereira AC, Tanuri AC, Fucciolo DK, Lobato ML et al. Tratamento anti-hipertensivo. Prescrição e custo de medicamentos. Pesquisa em hospital terciário. Arq Bras Cardiol 1998; 71:55-7.

4. Manolio TA, Cutler JA, Furberg CD, Psaty BM, Whelton PK, Applegate WB. Trends in pharmacologic managementofhypertension in the United States. Arch Intern Med 1995; I 55: 829-37.

5. The Sixth Report of the Joint National Committee on Prevention, Detection, Evaluation and Treatmente of High Blood Pressure. Arch Intern Med 1997; 157:2413-45.

6. Mion Jr D, Pierin AMG, Ignes EM et al. Conhecimentos, preferëncias e perfil dos hipetensos quanto ao tratamento farmacológico e não farmacológico. J Brass Nefrol 1995; 17: 229-36.

Artigo recebido: 01/06/2000 Aceito para publicação: 19/06/2001 